closer to home and the associated economic benefits of reducing paediatric retrieval to Tertiary Children's Hospital Intensive Care units.

Design Cross-sectional study.

Setting The Paediatric Unit (PU) at Campbelltown Hospital, Sydney; Australia. Campbelltown Hospital is a large outer metropolitan general hospital located $60 \mathrm{~km}$ from central Sydney. It services one of the largest paediatric populations in Sydney. Each year there are approximately 23000 paediatric emergency presentations and 3500 admissions. The PU has a 24-bed base and two COBs with the second COB being introduced in May 2018.

Patients 151 patients were admitted to a COB between January 2017 and December 2018. These acute patients met the severity criteria for a COB admission. They required higher nursing observations and medical interventions. Most patients were admitted for respiratory illness (127) requiring Humidified High Flow or CPAP. Other patients included drug infusions for diabetic ketoacidosis; seizures; and sepsis.

Results 136 patients (90\%) staying in a COB were successfully treated. This is more than the number of retrievals (119) within the 2 years. The COBs have potentially reduced the number of retrievals by more than $50 \%$.

COBs are equipped with a video conferencing link. This aided in the management of 14 patients (9.3\%) with tertiary teams remotely.

The COBs allowed patients' families to be treated closer to home. Of the 973 days which the 2 COBs were available, they were occupied for 218 days $(22.4 \%)$. Further demand was demonstrated by the additional increase in admissions with the start of second COB 96 (2018) compared to only 55 (2017) patients.

Reducing the number of retrievals resulted in significant economic savings with each retrieval costing an average of $\$ 1337.67$, equating to a total saving of $\$ 181923.12$ over 2 years.

Conclusion Paediatric Close Observation Beds play a vital role in managing severe sick children locally and the reduction in retrieval to Tertiary Children's Paediatric Intensive Care. This resulted in economic cost savings.

\section{P582 AUDIT ON ROLE OF BLOOD TESTS IN CHILDREN ADMITTED WITH COMMUNITY ACQUIRED PNEUMONIA}

1,2Pankaj Agrawal" ${ }^{*}{ }^{1}$ Muhammad Shoaib Iqbal, 'Asim Khan. 'Letterkenny University Hospital, Letterkenny, Ireland; ' ${ }^{2}$ Cork University Hospital, Cork, Ireland

\subsection{6/archdischild-2019-epa.916}

Background Community Acquired Pneumonia (CAP) is defined as a clinical diagnosis of pneumonia in a previously healthy child due to an infection which has been acquired outside hospital. It is the major cause of paediatric hospital admissions worldwide. Doing blood tests like CRP and white cell counts routinely in all the patients admitted with mild to moderate CAP is not recommended although in severe cases this can vary. Following the BTS guidelines, can prevent un-necessary burden on the laboratory and health care system and can reduce the duration of stay for patients admitted in Paediatric ward with mild to moderate community acquired pneumonia. It was observed that large number of children admitted with CAP routinely undergo blood investigations including white blood count \& CRP. We therefore decided to conduct an audit and implement the changes to prevent unnecessary blood investigations in children and to reduce the workload on Pathology department.

Objectives To establish if we are following the BTS guidelines for mild to moderate community acquired pneumonia regarding white cell count and CRP in children admitted to the Paediatric Ward in Letterkenny University Hospital.

Methods Data was collected from the Medical Records of children who have been admitted to the Paediatric Ward during the period 1st Dec 2016 to 28th Feb. 2017. Children more than one year of age who were diagnosed as mild to moderate community acquired pneumonia as per BTS guidelines were included in the study. Data was collected using a short questionnaire using the audit tool provided by the BTS guideline.

Results A total of 49 patients chart were obtained. Out of these, 18 patients were excluded from the study, and 31 patients met the diagnostic criteria for inclusion. Out of these 31 patients, 26 patients $(83.87 \%)$ had CRP done on them although it was documented that the children had community acquired pneumonia. All these 26 patients also had white cell count done on them as well.

Conclusion There is poor compliance as per BTS guidelines regarding routine CRP, White cell count in patients with mild to moderate community acquired pneumonia.

Recommendations

- British Thoracic society guidelines should be followed properly in managing patients with childhood community acquired pneumonia.

- Un-necessary CRP and white cell count should not be done in mild to moderate community acquired pneumonia

- To conduct regular teaching sessions on recent guidelines to keep updated all doctors working in Paediatric unit in Letterkenny University Hospital

\section{P583 AN UNCOMMON CAUSE OF SPONTANEOUS ECCHYMOSIS IN CHILDREN: ACUTE IDIOPATHIC THROMBOCYTOPENIC PURPURA}

Çiğdem El*, Mehmet Emin Çelikkaya. Mustafa Kemal University, Hatay, Turkey

10.1136/archdischild-2019-epa.917

Introduction The most common cause of acute onset thrombocytopenia in childhood is idiopathic thrombocytopenic purpura (ITP). The disease may seen in previously healthy children with only thrombocytopenia or with petechiae, purpura, ecchymosis and mucosal hemorrhage without clinical evidence. In this study, we aimed to evaluate the clinical presentation of ITP.

Methods Patients with acute ITP diagnosed between 2017 and 2018 in Mustafa Kemal University, Research Hospital, Department of Child Health and Diseases were retrospectively studied. Patients were evaluated according to age, gender, frequent seasons, referral complaints, history of previous infection and vaccination and ethnic group.

Results The clinical findings of the patients were as follows: petechia 100\% (n: 155), purpura 100\% (n: 155), ecchymosis $100 \%$ (n: 155), epistaxis 11,61 (n: 18), gingival bleeding 9,67: 15), gastrointestinal bleeding was $1.93 \%(\mathrm{n}: 3)$ and hematuria was $1.29 \%$ ( $\mathrm{n}: 2$ ). bone marrow biopsy was performed a patient with hepatosplenomegaly and 